\title{
Assessment of drainage techniques for evacuation of chronic subdural hematoma: a consecutive population-based comparative cohort study
}

\author{
*Kristin Sjåvik, MD, ${ }^{2}$ Jiri Bartek Jr., MD,,7 Lisa Millgård Sagberg, MSc, 3,4 \\ Marte Lødemel Henriksen, MD, ${ }^{2}$ Sasha Gulati, MD, PhD, ${ }^{3,4}$ Fredrik L. Ståhl, MD,1 \\ Helena Kristiansson, MD, ${ }^{1}$ Ole Solheim, MD, PhD, ${ }^{3,4}$ Petter Förander, MD, PhD, ${ }^{1}$ and \\ Asgeir Store Jakola, MD, PhD ${ }^{4-6}$
}

\begin{abstract}
'Department of Clinical Neuroscience, Karolinska Institutet, and Department of Neurosurgery, Karolinska University Hospital, Stockholm, Sweden; ${ }^{2}$ Department of Neurosurgery, University Hospital of North Norway, Troms $\emptyset$, Norway; ${ }^{3}$ Department of Neuroscience, Norwegian University of Science and Technology, Trondheim, Norway; ${ }^{4}$ Department of Neurosurgery, St. Olavs University Hospital, Trondheim, Norway; ${ }^{5}$ Department of Neurosurgery, Sahlgrenska University Hospital, Gothenburg, Sweden; ${ }^{6}$ Institute of Neuroscience and Physiology, University of Gothenburg, Sahlgrenska Academy, Gothenburg, Sweden; and ${ }^{7}$ Department of Neurosurgery, Copenhagen University Hospital Rigshospitalet, Copenhagen, Denmark
\end{abstract}

OBJECTIVE Surgery for chronic subdural hematoma (CSDH) is one of the most common neurosurgical procedures. The benefit of postoperative passive subdural drainage compared with no drains has been established, but other drainage techniques are common, and their effectiveness compared with passive subdural drains remains unknown.

METHODS In Scandinavian population-based cohorts the authors conducted a consecutive, parallel cohort study to compare different drainage techniques. The techniques used were continuous irrigation and drainage (CID cohort, $n=$ 166), passive subdural drainage (PD cohort, $n=330)$, and active subgaleal drainage ( $A D$ cohort, $n=764)$. The primary end point was recurrence in need of reoperation within 6 months of index surgery. Secondary end points were complications, perioperative mortality, and overall survival. The analyses were based on direct regional comparison (i.e., surgical strategy).

RESULTS Recurrence in need of surgery was observed in 18 patients (10.8\%) in the CID cohort, in 66 patients (20.0\%) in the PD cohort, and in 85 patients $(11.1 \%)$ in the AD cohort $(p<0.001)$. Complications were more common in the CID cohort $(14.5 \%)$ compared with the $P D(7.3 \%)$ and $A D(8.1 \%)$ cohorts $(p=0.019)$. Perioperative mortality rates were similar between cohorts $(p=0.621)$. There were some differences in baseline and treatment characteristics possibly interfering with the above-mentioned results. However, after adjusting for differences in baseline and treatment characteristics in a regression model, the drainage techniques were still significantly associated with clinical outcome $(p<0.001$ for recurrence, $p=0.017$ for complications).

CONCLUSIONS Compared with the AD cohort, more recurrences were observed in the PD cohort and more complications in the CID cohort, also after adjustment for differences at baseline. Although the authors cannot exclude unmeasured confounding factors when comparing centers, $A D$ appears superior to the more common PD.

Clinical trial registration no.: NCT01930617 (clinicaltrials.gov)

https://thejns.org/doi/abs/10.3171/2016.12.JNS161713

KEY WORDS chronic subdural hematoma; drainage; treatment outcomes; trauma

ABBREVIATIONS $\mathrm{AD}=$ active subgaleal drain $; \mathrm{CCl}=$ Charlson Comorbidity Index $\mathrm{Cl}=$ confidence interval; $\mathrm{CID}=$ continuous irrigation and drainage; $\mathrm{CSDH}=\mathrm{chronic}$ subdural hematoma; $\mathrm{HR}=$ hazard ratio; $\mathrm{PD}=$ passive subdural drain; $\mathrm{RCT}=$ randomized controlled trial.

SUBMITTED July 1, 2016. ACCEPTED December 21, 2016.

INCLUDE WHEN CITING Published online June 23, 2017; DOI: 10.3171/2016.12.JNS161713.

* Drs. Sjåvik and Bartek contributed equally to this work. 
$\mathrm{E}$ VACUATION of chronic subdural hematoma (CSDH) is one of the most common neurosurgical procedures. The number of patients with $\mathrm{CSDH}$ is expected to increase as the oldest segment of the population continues to grow, and according to the prognosis for $2030, \mathrm{CSDH}$ is expected to be the most common cranial neurosurgical condition in the US., ${ }^{3,14}$ Surgical techniques vary, but following the landmark trial from Santarius et al. in 2009, the use of postoperative drainage is now the norm. ${ }^{21}$ However, recurrence rates are high, and ways to achieve further improvements should be sought.

Today, common treatment variants include a twist-drill craniostomy, burr-hole procedure with passive subdural drainage, subgaleal (or subperiosteal) active (suction) drainage, and continuous irrigation..$^{2,5,7-9,21}$ There is no evidence supporting the superiority of one specific drainage technique, and adequately powered comparative studies evaluating the different drainage techniques after burrhole evacuation are lacking. ${ }^{11,14}$

The aim of this population-based, retrospective, comparative cohort study was to compare recurrences after CSDH burr-hole surgery in regions served by neurosurgical centers using different postoperative drainage techniques.

\section{Methods}

\section{Ethics and Approvals}

The study was approved by the Stockholm regional ethical review board in Sweden and the Regional Committee for Medical and Health Research Ethics in Central Norway. The study was registered in clinicaltrials.gov (no. NCT01930617) prior to data collection. Reporting is consistent with the STROBE (Strengthening the Reporting of Observational Studies in Epidemiology) statement.

\section{Scandinavian Health Care System and Treatment Centers}

The health care system in Norway and Sweden is divided into different geographical regions with compliant referral patterns for intracranial surgery within these regions. A patient with a CSDH in the greater Stockholm region will be referred to and cared for at the neurosurgical department at Karolinska University Hospital. Similarly, patients with CSDH in Northern Norway and Central Norway will be referred to the University Hospital of North Norway and St. Olavs University Hospital, respectively. Because no private health care alternative exists for patients with CSDH and a strict regional referral is used, the risk of referral bias is practically eliminated. Data from Statistics Norway (http://www.ssb.no) and Statistics Sweden (http://www.scb.se) were used to estimate the mean population of the respective hospital catchment areas during the study period.

\section{Patient Population}

All adult patients (18 years or older) treated with evacuation of primary CSDH between January 1, 2005, and December 31, 2010, at the neurosurgery departments of Karolinska University Hospital (Stockholm, Sweden), the University Hospital of North Norway (Troms $\varnothing$, Norway) and St. Olavs University Hospital (Trondheim, Norway) were identified using the hospitals' patient administrative databases and operating room logs. Patients who underwent any other form of intracranial surgery during the last 6 months prior to CSDH intervention and those having CSDH in relation to arachnoid cysts were not included. In total, 1260 patients were included. As noted in Table 1, there were 166 patients (13\%) who underwent operations for CSDH at University Hospital of North Norway, 330 patients $(26 \%)$ at St. Olavs University Hospital, and 764 patients (61\%) at Karolinska University Hospital during the inclusion period. The incidence rates of surgical intervention for CSDH were 7.8 per 100,000 people per year at University Hospital of North Norway, 10.9 at St. Olavs University Hospital, and 8.0 at Karolinska University Hospital $(\mathrm{p}=0.034)$. The age- and sex-adjusted incidence rates were $7.5,10.3$, and 8.4 per 100,000 people per year, respectively $(\mathrm{p}=0.096)$.

\section{Treatment Regimens}

All 3 centers routinely used single burr holes and irrigate intraoperatively, but the use of drainage systems and postoperative care differed. At 1 center, a continuous inflow and outflow irrigation was established (Charriere no. 14 drain for outflow, Nelaton catheter no. 10 for inflow of irrigation fluid, consisting of sterile saline with gentamicin added at a concentration of $10 \mathrm{mg} / 1000 \mathrm{ml}$ ), and typically continued for 24 hours, or until clear outflow fluid was noted, i.e., the continuous irrigation and drainage (CID) cohort. ${ }^{9}$ The second center used passive subdural drains (external ventricular drain, Medtronic barium-impregnated ventricular catheter, $35 \mathrm{~cm}$ in length, $2.8-\mathrm{mm}$ outer diameter, $1.5-\mathrm{mm}$ inner diameter), that was placed at the end of surgery and kept overnight, usually for 12-18 hours, i.e., the passive subdural drain (PD) cohort. At the third center, subgaleal active (suction) drainage was used (10-Fr catheter) for 24 hours, i.e., the active subgaleal drain (AD) cohort. The different drainage systems are illustrated in Fig. 1. In the CID and AD cohorts, immobilization during drainage was strictly enforced, and patients were kept in intermediate ward units. In the PD cohort there was no uniform practice concerning mobilization, but patients were typically in an intermediate/postoperative ward unit 2-4 hours postoperatively, after which they were transferred to a regular ward unit. Importantly, each treatment method was well established and uniform at the involved centers in the study period.

\section{Study Variables}

We defined the index operation as the first surgical procedure on the affected side. Bilateral hematomas were registered as 1 index operation if both sides were treated as part of the same procedure. A recurrent CSDH was defined as a same-sided CSDH recurrence treated with surgery within 6 months of the index operation. In the cases in which a 1-sided index operation was followed by a bilateral recurrent procedure (i.e., 1 recurrent side and 1 untreated side), the patient was still registered as having only 1 index operation and 1 reoperation.

Patient and treatment characteristics were retrospectively retrieved from medical records. Radiological variables were extracted from the respective hospitals' picture 
TABLE 1. Baseline characteristics of the study population

\begin{tabular}{|c|c|c|c|c|}
\hline Variable & CID Cohort & PD Cohort & AD Cohort & p Value \\
\hline No. of patients (\%) & $166(13)$ & $330(26)$ & $764(61)$ & \\
\hline Age- \& sex-adjusted incidence rates* & 7.5 & 10.3 & 8.4 & 0.096 \\
\hline Mean age in yrs (SD) & $73(11)$ & $74(13)$ & $74(13)$ & 0.470 \\
\hline $\mathrm{CCl}>1(\%)$ & $58(34.9)$ & $99(30.0)$ & $270(35.3)$ & 0.220 \\
\hline Males (\%) & $111(66.9)$ & $249(75.5)$ & $518(67.8)$ & 0.029 \\
\hline Level of independence (\%) & & & & 0.093 \\
\hline Independent & $129(77.7)$ & $237(72.0)$ & $571(74.9)$ & \\
\hline Home w/ care & $17(10.2)$ & $60(18.2)$ & $130(17.1)$ & \\
\hline Residential/nursing home & $20(12.0)$ & $33(10.0)$ & $61(8.0)$ & \\
\hline Missing & 0 & 1 & 2 & \\
\hline \multicolumn{5}{|l|}{ Preop symptoms (\%) } \\
\hline Paresis & $89(53.6)$ & $127(38.5)$ & $334(43.7)$ & 0.006 \\
\hline Dysphasia & $52(31.3)$ & $90(27.3)$ & $180(23.6)$ & 0.081 \\
\hline Headache & $80(48.2)$ & $139(42.1)$ & $282(36.9)$ & 0.016 \\
\hline Vomiting & $12(7.2)$ & $27(8.2)$ & $36(4.7)$ & 0.064 \\
\hline GCS score $(\%)$ & & & & 0.010 \\
\hline $3-8$ & $3(1.8)$ & $15(4.5)$ & $21(2.8)$ & \\
\hline $9-12$ & $21(12.7)$ & $21(6.4)$ & $43(5.8)$ & \\
\hline $13-15$ & $142(85.5)$ & $294(89.1)$ & $682(91.4)$ & \\
\hline Missing & 0 & 0 & 18 & \\
\hline Hematoma density & & & & $<0.001$ \\
\hline Hypodense \& mixed density & $128(84.8)$ & $218(79.3)$ & $523(70.4)$ & \\
\hline Iso- \& hyperdense & $23(15.2)$ & $57(20.7)$ & $220(29.6)$ & \\
\hline Missing & 15 & 55 & 21 & \\
\hline Mean largest midline shift in mm (SD) & $8.7(5.1)$ & $7.3(4.8)$ & $7.8(4.5)$ & 0.009 \\
\hline Missing & 16 & 40 & 18 & \\
\hline Platelet inhibitor (\%) & $52(31.5)$ & $92(27.9)$ & $193(25.4)$ & 0.239 \\
\hline Missing & 1 & 0 & 3 & \\
\hline Anticoagulants (\%) & $39(23.6)$ & $63(19.1)$ & $110(14.5)$ & 0.008 \\
\hline Missing & 1 & 0 & 4 & \\
\hline
\end{tabular}

GCS = Glasgow Coma Scale.

* Per 100,000 people per year.

archiving and communication systems. A CSDH in this study was not restricted to hypodense hematomas only, but also hematomas with higher densities and including acute elements as reported by others, but where burr-hole surgery was a reasonable option. ${ }^{18,23}$ The Charlson Comorbidity Index (CCI) was used to assess comorbidity. ${ }^{6}$
The Landriel Ibañez classification was used to evaluate complications within 30 days postoperatively. ${ }^{16}$ However, recurrent CSDH within this 30-day period was not considered a surgical complication, because recurrence is part of the natural course of the diagnosis, and most importantly, the primary end point of the study.
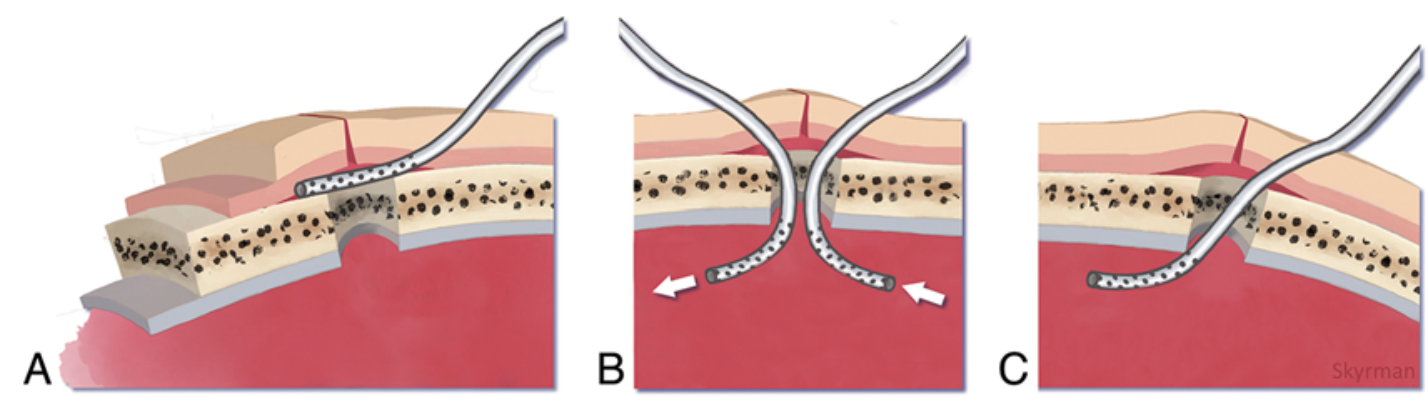

FIG. 1. Illustration of the 3 different drainage techniques. A: AD technique with active/suction drainage placed in a subgaleal position overlying the burr hole. B: CID technique with active inflow and passive outflow drainage placed in the subdural space. C: $\mathrm{PD}$ technique with passive drainage in the subdural space. Copyright Simon Skyrman. Published with permission. Figure is available in color online only. 


\section{End Points}

The prespecified primary end point was the proportion of reoperations for same-sided CSDH within 6 months, analyzed according to treatment policy (i.e., making regional comparisons).

The prespecified secondary end points were overall survival, perioperative mortality (within 30 days), and surgical complications, according to the Landriel Ibañez classification. These end points were analyzed with respect to regional treatment policy and not actual treatment.

\section{Statistical Analysis}

All analyses were performed using SPSS (version 21.0, IBM Corp.). The statistical significance level was set to $\mathrm{p}$ $\leq 0.05$. All tests were 2 -sided. Central tendencies are presented as means \pm SDs. Categorical data were analyzed using Pearson's chi-square test. Comparisons of means between departments were analyzed using ANOVA statistics. Overall survival is presented as Kaplan-Meier curves and compared using a log-rank test. Age- and sex-matched incidence rates were calculated using the 2008 Norwegian adult population (18 years and older) census as a reference (available at Statistics Norway, http://www.ssb.no). The post hoc adjusted logistic regression analyses were created due to baseline imbalances between groups.

\section{Results}

Baseline patient characteristics for the 3 groups are presented and compared in Table 1. There were some differences between the groups with respect to sex $(p=0.029)$, preoperative symptoms including paresis $(\mathrm{p}=0.006)$ and headaches $(p=0.016)$, the level of consciousness before treatment $(\mathrm{p}=0.01)$, hematoma density $(\mathrm{p}<0.001)$, magnitude of midline shift ( $\mathrm{p}=0.009)$, and the use of anticoagulants $(\mathrm{p}=0.008)$.

As noted in Table 2, treatment was in accordance with the institutional policy in the vast majority of patients. Policy adherence was still significantly different between institutions, with 147/166 (88.6\%) in the CID cohort, 324/330 (98.2\%) in the PD cohort, and 760/764 (99.5\%) in the AD cohort $(\mathrm{p}<0.001)$ treated according to institutional policy. Further treatment details are presented in Table 2.

\section{Primary End Point}

For the entire study population, recurrent CSDH in need of surgery was detected after 169 operations (13.4\%). Treatment outcome is presented for the different groups in Table 3. Recurrence occurred following 18 (10.8\%) of 166 operations in the CID cohort, 66 (20.0\%) of 330 operations in the PD cohort, and 85 (11.1\%) of 764 operations in the AD cohort $(\mathrm{p}<0.001)$. Data on the primary end point are presented in Fig. 2. The CID cohort was least compliant, and we explored the proportion of recurrence when using this technique in an as-treated analysis. The risk of recurrence when actually treated with CID was $10.2 \%$ (15/147).

\section{Secondary End Points}

Treatment outcomes are summarized and presented in Table 3. More complications were observed in the CID cohort (14.5\%) compared with the PD (7.3\%) and AD (8.1\%) cohorts $(\mathrm{p}=0.019)$. When analyzing moderate to severe complications (Landriel Ibañez Grades II-IV), with 6.0\% complications in the CID cohort compared with $3.0 \%$ and $4.6 \%$ in $\mathrm{PD}$ and $\mathrm{AD}$ cohorts, respectively, the statistically significant difference disappeared $(\mathrm{p}=0.279)$. Gentamicin in the irrigation as used in the CID cohort was not associated with a lower proportion of locoregional infection ( $1.8 \%$ vs $0.6 \%$ and $1.6 \%, \mathrm{p}=0.384)$. On the other hand, there was no increased risk of seizures or other suspected toxicity with gentamicin use $(1.2 \%$ vs $0.6 \%$ and $1.2 \%, \mathrm{p}=$ 0.672). Perioperative mortality rates within 30 days after surgery were $3.0 \%$ in the CID cohort, $4.3 \%$ in the PD cohort, and $3.1 \%$ in the AD cohort ( $\mathrm{p}=0.621)$. The overall survival rate was similar between regions (Table 3, Fig. 1).

\section{Post Hoc Regression Analyses}

In post hoc regression analyses adjusting for the differences in baseline factors (hematoma density, midline shift, surgical procedure, age, and sex), the type of drainage technique continued to be associated with surgery for

TABLE 2. Treatment characteristics

\begin{tabular}{|c|c|c|c|c|}
\hline Treatment Variable & CID Cohort (\%) & PD Cohort (\%) & AD Cohort (\%) & $p$ Value \\
\hline Treatment according to policy & $147(88.6)$ & $324(98.2)$ & $760(99.5)$ & $<0.001$ \\
\hline Actual treatment & & & & $<0.001$ \\
\hline Burr-hole subdural passive & $11(6.6)$ & $324(98.2)$ & $1(0.1)$ & \\
\hline Burr-hole continuous & $147(88.6)$ & 0 & 0 & \\
\hline Burr-hole no drain & $7(4.2)$ & $4(1.2)$ & 0 & \\
\hline Burr-hole subgaleal active & $1(0.6)$ & 0 & $760(99.5)$ & \\
\hline Craniotomy & 0 & $2(0.6)$ & $3(0.4)$ & \\
\hline General anesthesia & $31(19.0)$ & $13(3.9)$ & $33(4.3)$ & $<0.001$ \\
\hline Missing & 3 & 0 & 0 & \\
\hline Side of surgery & & & & 0.685 \\
\hline Rt & $63(38.4)$ & $116(37.1)$ & $279(37.4)$ & \\
\hline Lt & $74(45.1)$ & $133(42.5)$ & $340(45.6)$ & \\
\hline Bilat & $27(16.5)$ & $64(20.4)$ & $126(16.9)$ & \\
\hline Missing & 2 & 17 & 19 & \\
\hline
\end{tabular}




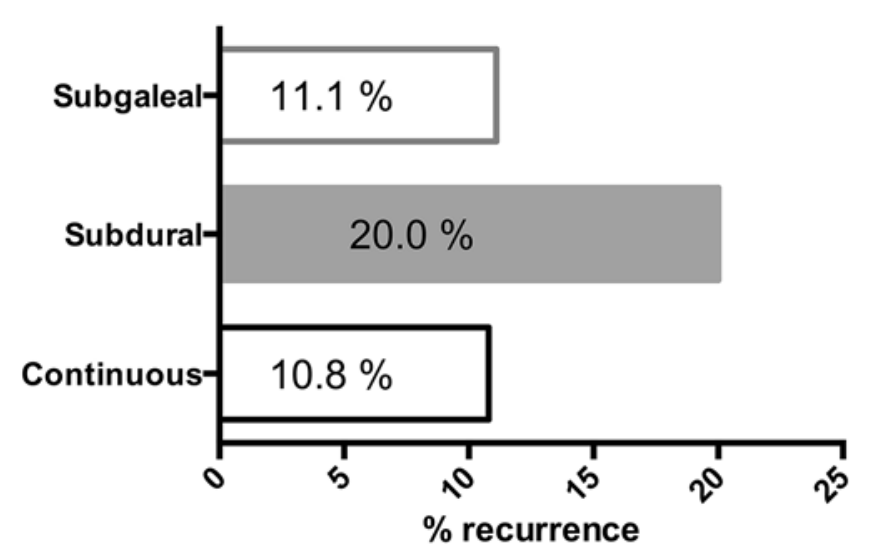

FIG. 2. Bar graph showing the results of the primary end point (proportion of recurrences between groups). There was a statistically significant difference between groups $(p<0.001)$.

recurrence $(\mathrm{p}<0.001$, Table 4). A similar analysis using the same variables demonstrated that type of surgical procedure continued to be associated with complications after adjustment (hazard ratio [HR] 1.94, 95\% confidence interval $[\mathrm{CI}] 1.13-3.35, \mathrm{p}=0.017)$.

\section{Discussion}

This consecutive population-based retrospective parallel cohort study shows that AD and CID were associated with a lower chance of reoperation due to CSDH recurrence compared with only PD postoperatively. Further, more complications were observed in the cohort treated with CID. There were no differences in mortality rates and overall survival between cohorts. The differences in outcomes across cohorts remained following adjustment for baseline characteristics.
TABLE 4. Adjusted binary logistic regression analyses*

\begin{tabular}{lccc}
\hline \multicolumn{1}{c}{ Variable } & HR & $95 \% \mathrm{Cl}$ & p Value \\
\hline Midline shift (per increasing mm) & 1.00 & $0.97-1.04$ & 0.83 \\
\hline Male (vs female) & 2.20 & $1.41-3.43$ & $<0.001$ \\
\hline Age (per increasing yr) & 1.00 & $0.99-1.02$ & 0.96 \\
\hline PD (vs other techniques) & 2.00 & $1.39-2.89$ & $<0.001$ \\
\hline Hypodensity (vs other) & 0.95 & $0.63-1.44$ & 0.82 \\
\hline Anticoagulant (vs no anticoagulant) & 1.25 & $0.82-1.92$ & 0.30 \\
\hline GCS score (per increasing point) & 0.96 & $0.88-1.05$ & 0.34 \\
\hline
\end{tabular}

Boldface type indicates statistical significance.

* Significant imbalances at baseline as noted in Table 1 were selected as univariates into the model, except symptoms because they are likely related to GCS score.

Because there were large differences in recurrence rates, we believe that postoperative drainage technique matters. However, there were differences in incidence rates across the 3 geographical regions, and we cannot exclude the possibility that regional differences in treatment indications, follow-up routines, or indications for reoperations may have affected our findings. Since incidence rates of surgery for CSDH were higher in the PD region (although nonsignificantly so after adjusting for age and sex), this may suggest a more liberal indication for surgery, which may also be true for reoperations. This may, however, be a factor in both directions because presumably more patients with smaller CSDHs and favorable prognoses may have been offered surgery, but also more patients could have been offered repeated surgery based on vague symptoms. In the PD cohort, patients were routinely screened 4 weeks postoperatively with a visit in the outpatient clinic and a CT scan, while CT controls were performed based on clinical symptoms in the CID and

TABLE 3. Secondary end points

\begin{tabular}{|c|c|c|c|c|}
\hline Secondary End Point & CID Cohort (\%) & PD Cohort (\%) & AD Cohort (\%) & $p$ Value \\
\hline Reop & $18(10.8)$ & $66(20.0)$ & 85 (11.1) & $<0.001$ \\
\hline Time to reop & & & & 0.943 \\
\hline$<1 \mathrm{mo}$ & $11(61.1)$ & $43(65.2)$ & $53(62.4)$ & \\
\hline $1-2$ mos & $5(27.8)$ & $19(28.8)$ & $24(28.2)$ & \\
\hline 2-6 mos & $2(11.1)$ & $4(6.1)$ & $8(9.4)$ & \\
\hline Reason for reop & & & & 0.726 \\
\hline Symptoms \& imaging & $17(94.4)$ & $61(92.4)$ & $73(86.9)$ & \\
\hline Symptoms only & 0 & $3(4.5)$ & $7(8.3)$ & \\
\hline Imaging only & $1(5.6)$ & $1(1.5)$ & $4(4.8)$ & \\
\hline Missing & 0 & 0 & 1 & \\
\hline Complication & $24(14.5)$ & $24(7.3)$ & $62(8.1)$ & 0.019 \\
\hline Missing & 0 & 0 & 1 & \\
\hline Landriel Ibañez Grade $\geq \|$ & $10(6.0)$ & $10(3.0)$ & $35(4.6)$ & 0.279 \\
\hline Missing & 0 & 0 & 1 & \\
\hline \multicolumn{5}{|l|}{ Mortality } \\
\hline 30 days & $5(3.0)$ & $14(4.3)$ & $24(3.1)$ & 0.621 \\
\hline 90 days & $10(6.0)$ & $24(7.3)$ & $44(5.8)$ & 0.627 \\
\hline Death in follow-up & $55(33.1)$ & $101(30.7)$ & $206(27.0)$ & 0.191 \\
\hline Missing & 0 & 1 & 1 & \\
\hline
\end{tabular}


AD cohorts. Importantly, the indications for reoperations were not different between cohorts based on our retrospective review, as patients had to present with symptoms in addition to radiological findings. Furthermore, the timing of repeated surgery was similar, suggesting that the immediate postoperative care in the other regions was sufficient as well. The differences in baseline characteristics were not systematically in disfavor of the PD cohort, and the significant impact of drainage technique was sustained after adjusting for baseline imbalances. The difference in recurrences between cohorts was large, and although the above-mentioned may have influenced our results, at least in part, it appears unlikely that our findings can be explained simply by indication and detection bias.

Our findings in the PD cohort need to be interpreted in light of the proportion of recurrences reported by Santorius et al. (9.3\%) that established the need for postoperative drainage. ${ }^{21}$ Prior to analysis we expected the results to be comparable, as the Santorius study also used a subdural drainage and assessed recurrence within 6 months of index surgery. Compared with our study, several important differences not merely related to the drainage technique are present, forcing us to adjust the strength of our recommendations. First, the Santorius et al. study used 2 burr holes instead of 1, which may facilitate CSDH evacuation. ${ }^{24}$ Second, the drain was kept in place for 48 hours, compared with less standardized drainage time in the subdural group in our study, which often was surgery in the evening and removal of the drainage system the following morning. The prolonged drainage time may also facilitate CSDH evacuation. ${ }^{26}$ In fact, in terms of recurrence, the subdural group in our study is comparable to the "no drain" group in the Santorius study (24\% recurrences in need of surgery), implying an inadequate evacuation of the subdural fluid in this cohort.

The hospitals using techniques other than passive subdural drainage techniques had standardized postoperative routines and outcomes comparable to those in the study of Santorius et al. ${ }^{21}$ Thus, our data strongly suggest that other drainage techniques are not inferior to the traditional passive subdural drainage technique. The CID cohort performed worse than expected (10.8\% recurrence rate) compared with a previous, smaller study from the same center demonstrating recurrence in need of surgery in $2.6 \%$ of patients. ${ }^{9}$ Gazzerri and colleagues have published the largest series to date on subgaleal drainage through a $3-\mathrm{cm}$ minicraniotomy and prolonged drainage time (i.e., 48-72 hours) and reported $7.6 \%$ recurrence in need of a repeated procedure, a finding comparable to our subgaleal cohort using a single burr hole and 24-hour drainage (10.8\%). ${ }^{8}$ Moreover, the highly selected patients participating in randomized trials commonly fare better than patients treated in routine clinical practice. ${ }^{4}$ In addition, the Hawthorne effect may also account for some of the difference in effectiveness of passive subdural drainage observed between our PD cohort and the Santorius study. ${ }^{10,20}$ In the PD cohort patients were routinely screened with a CT scan at follow-up after index surgery, while this was replaced by a clinical follow-up with CT control "as needed" in the CID and AD cohorts. Concerning the differences in the Santorius study compared with our PD cohort, we simply conclude that the use of PD for short drainage times $(<24$ hours) through a single burr hole seems questionable.

We found increased morbidity in the CID cohort. This cohort may in some cases have experienced delayed mobilization beyond 24 hours, and general anesthesia was more common in this cohort. The association between increased morbidity and delayed mobilization is suggested by a previous case-control study, ${ }^{15}$ although conflicting reports exist on this matter. ${ }^{1}$ Two recent studies reported that general anesthesia was associated with higher morbidity and longer hospital stays than sedation combined with local anesthesia, whereas previous studies did not report such differences. Although general anesthesia was more common in the CID cohort, there were no differences in perioperative mortality between cohorts.

With the exception of a randomized controlled trial (RCT) reporting that postoperative subdural drainage was better than no drainage, ${ }^{21}$ there is a lack of high-quality evidence in CSDH management. ${ }^{17,22}$ Another RCT did not replicate the benefits of a drainage system, but the study was severely underpowered..$^{13}$ As blinding of personnel and participants in neurosurgical RCTs is difficult and sometimes impossible, pragmatic comparative studies may complement RCTs. ${ }^{12,19}$ We present a comparative population-based study of 1260 patients, aiming to clarify the risks and benefits of 3 different treatment regimes for CSDH. The sample size allows for reliable assessment of recurrences, perioperative morbidity, and mortality. This approach may be a way to improve the level of evidence in neurosurgical research compared with the common single-institution case series. ${ }^{25}$

Limitations inherent to retrospective assessment are present in this study, but the clinical relevance of recurrence and mortality remain indisputable. As mentioned earlier, detection bias may have been introduced by different policies with respect to postoperative imaging between cohorts. Although this may have influenced the proportion of recurrences in this region to some extent, given the magnitude of effect detection, bias alone is unlikely to fully explain the observed difference. Also, the difference in outcomes may be at least in part due to the fact that each type of procedure was performed at different hospitals and by different surgical teams. Further, differences in postoperative management may also have contributed to this difference with less standardized and shorter hospital stays in the subdural group. The major strengths of this study are a very high compliance with treatment strategy in combination with the population-based approach and low rates of missing data.

\section{Conclusions}

Active subgaleal drainage and continuous irrigation/ drainage were superior to passive subdural drainage with respect to recurrences in this population-based, retrospective comparative cohort study. A higher complication rate was observed in the group with CID, possibly a combination of a more extensive procedure, a more frequent use of general anesthesia, and a longer immobilization period/ bed rest. Mortality rates and overall survival were similar between groups. In conclusion, our data indicate that the 
least invasive surgical method, using subgaleal drainage technique for 24 hours postoperatively, appears more effective than passive subdural drainage as used in this study and safer than CID.

\section{Acknowledgments}

We would like to thank Dr. Simon Skyrman for his invaluable graphic support with Fig. 1 in this manuscript. Kristin Sjåvik received research funding from Medical Imaging Lab, Norwegian University for Science and Technology (Trondheim, Norway).

\section{References}

1. Abouzari M, Rashidi A, Rezaii J, Esfandiari K, Asadollahi $\mathrm{M}$, Aleali $\mathrm{H}$, et al: The role of postoperative patient posture in the recurrence of traumatic chronic subdural hematoma after burr-hole surgery. Neurosurgery 61:794-797, 2007

2. Almenawer SA, Farrokhyar F, Hong C, Alhazzani W, Manoranjan B, Yarascavitch B, et al: Chronic subdural hematoma management: a systematic review and meta-analysis of 34,829 patients. Ann Surg 259:449-457, 2014

3. Balser D, Farooq S, Mehmood T, Reyes M, Samadani U: Actual and projected incidence rates for chronic subdural hematomas in United States Veterans Administration and civilian populations. J Neurosurg 123:1209-1215, 2015

4. Braunholtz DA, Edwards SJ, Lilford RJ: Are randomized clinical trials good for us (in the short term)? Evidence for a "trial effect". J Clin Epidemiol 54:217-224, 2001

5. Chari A, Kolias AG, Santarius T, Bond S, Hutchinson PJ: Twist-drill craniostomy with hollow screws for evacuation of chronic subdural hematoma. J Neurosurg 121:176-183, 2014

6. Charlson ME, Pompei P, Ales KL, MacKenzie CR: A new method of classifying prognostic comorbidity in longitudinal studies: development and validation. J Chronic Dis 40:373383, 1987

7. Ducruet AF, Grobelny BT, Zacharia BE, Hickman ZL, DeRosa PL, Andersen KN, et al: The surgical management of chronic subdural hematoma. Neurosurg Rev 35:155-169, 2012 (Erratum in Neurosurg Rev 38:771, 2015)

8. Gazzeri R, Galarza M, Neroni M, Canova A, Refice GM, Esposito S: Continuous subgaleal suction drainage for the treatment of chronic subdural haematoma. Acta Neurochir (Wien) 149:487-493, 2007

9. Hennig R, Kloster R: Burr hole evacuation of chronic subdural haematomas followed by continuous inflow and outflow irrigation. Acta Neurochir (Wien) 141:171-176, 1999

10. Holden JD: Hawthorne effects and research into professional practice. J Eval Clin Pract 7:65-70, 2001

11. Ivamoto HS, Lemos HP Jr, Atallah AN: Surgical treatments for chronic subdural hematomas: a comprehensive systematic review. World Neurosurg 86:399-418, 2016

12. Jakola AS, Myrmel KS, Kloster R, Torp SH, Lindal S, Unsgård $\mathrm{G}$, et al: Comparison of a strategy favoring early surgical resection vs a strategy favoring watchful waiting in lowgrade gliomas. JAMA 308:1881-1888, 2012

13. Javadi A, Amirjamshidi A, Aran S, Hosseini SH: A randomized controlled trial comparing the outcome of burr-hole irrigation with and without drainage in the treatment of chronic subdural hematoma: a preliminary report. World Neurosurg 75:731-736, 620-623, 2011

14. Kolias AG, Chari A, Santarius T, Hutchinson PJ: Chronic subdural haematoma: modern management and emerging therapies. Nat Rev Neurol 10:570-578, 2014

15. Kurabe S, Ozawa T, Watanabe T, Aiba T: Efficacy and safety of postoperative early mobilization for chronic subdural hematoma in elderly patients. Acta Neurochir (Wien) 152:1171-1174, 2010

16. Landriel Ibañez FA, Hem S, Ajler P, Vecchi E, Ciraolo C, Baccanelli M, et al: A new classification of complications in neurosurgery. World Neurosurg 75:709-715, 604-611, 2011

17. Liu W, Bakker NA, Groen RJ: Chronic subdural hematoma: a systematic review and meta-analysis of surgical procedures. J Neurosurg 121:665-673, 2014

18. Nakaguchi H, Tanishima T, Yoshimasu N: Factors in the natural history of chronic subdural hematomas that influence their postoperative recurrence. J Neurosurg 95:256-262, 2001

19. Nerland US, Jakola AS, Solheim O, Weber C, Rao V, Lønne $\mathrm{G}$, et al: Minimally invasive decompression versus open laminectomy for central stenosis of the lumbar spine: pragmatic comparative effectiveness study. BMJ 350:h1603, 2015

20. Parsons HM: What happened at Hawthorne?: New evidence suggests the Hawthorne effect resulted from operant reinforcement contingencies. Science 183:922-932, 1974

21. Santarius T, Kirkpatrick PJ, Ganesan D, Chia HL, Jalloh I, Smielewski P, et al: Use of drains versus no drains after burr-hole evacuation of chronic subdural haematoma: a randomised controlled trial. Lancet 374:1067-1073, 2009

22. Shapey J, Glancz LJ, Brennan PM: Chronic subdural haematoma in the elderly: is it time for a new paradigm in management? Curr Geriatr Rep 5:71-77, 2016

23. Stanišić M, Hald J, Rasmussen IA, Pripp AH, Ivanović J, Kolstad F, et al: Volume and densities of chronic subdural haematoma obtained from CT imaging as predictors of postoperative recurrence: a prospective study of 107 operated patients. Acta Neurochir (Wien) 155:323-333, 2013

24. Taussky P, Fandino J, Landolt H: Number of burr holes as independent predictor of postoperative recurrence in chronic subdural haematoma. Br J Neurosurg 22:279-282, 2008

25. Yarascavitch BA, Chuback JE, Almenawer SA, Reddy K, Bhandari M: Levels of evidence in the neurosurgical literature: more tribulations than trials. Neurosurgery 71:11311138,2012

26. Yu GJ, Han CZ, Zhang M, Zhuang HT, Jiang YG: Prolonged drainage reduces the recurrence of chronic subdural hematoma. Br J Neurosurg 23:606-611, 2009

\section{Disclosures}

The authors report no conflict of interest concerning the materials or methods used in this study or the findings specified in this paper.

\section{Author Contributions}

Conception and design: Bartek, Sjåvik, Jakola. Acquisition of data: Bartek, Sjåvik, Sagberg, Henriksen, Ståhl, Kristiansson. Analysis and interpretation of data: Bartek, Sjåvik, Sagberg, Henriksen, Gulati, Jakola. Drafting the article: Bartek. Critically revising the article: Bartek, Sjåvik, Gulati, Solheim, Förander, Jakola. Reviewed submitted version of manuscript: Bartek, Sjåvik, Solheim, Förander, Jakola. Approved the final version of the manuscript on behalf of all authors: Bartek, Statistical analysis: Bartek. Sjåvik, Jakola. Administrative/technical/material support: Bartek, Sjåvik, Jakola. Study supervision: Solheim, Förander, Jakola.

\section{Correspondence}

Jiri Bartek Jr., Department of Neurosurgery, Karolinska University Hospital, Stockholm 17176, Sweden. email: jiri.bartek@ karolinska.se. 